\title{
The Relation Between Septic Shock and Glycemic Control In Critically Ill Patients In Benha University Hospitals
}

\author{
K.S.Moselhey ${ }^{1}$, M.E.Ibrahim ${ }^{2}$, B.M.Aglan ${ }^{3}$ and W.M.El-Said ${ }^{1}$ \\ ${ }^{1}$ Neurology, Dept., Faculty of Medicine, Benha Univ., Benha, Egypt \\ ${ }^{2}$ Internal Medicine, Dept., Faculty of Medicine, Benha Univ., Benha, Egypt \\ ${ }^{3}$ Cardiothorathic Surgery, Dept., Faculty of Medicine, Benha Univ., Benha, Egypt
}

E.Mail:wmzayed1@gmail.com

\begin{abstract}
Hyperglycemia is common event in critical unit including patients with or without history of diabetes . in patients with septic shock, blood glucose level easily elevated by complex of pathophysiological mechanisms, we investigate the effect of normalization of blood glucose in septic shock patients including the morbidity, the mortality, the length of icu stay and the time on mechanical ventilation We included 60 patients divided into two groups.Group1:consist of 30 patients was applied to intensive glycemic control. Group 2: consist of 30 patients was applied to conventional glycemic control. We found that strict normalization of blood glucose have favourable outcome than conventional control in morbidity but not mortality. $20 \%$ in group 1 needed hemodialysis and $80 \%$ of patients required hemodialysis in group 2 which is statistically highly significant. Mortality rate $60 \%$ in group 1 and $80 \%$ in group 2 which is statistically insignificant. We found that intensive glycemic control have more favourable effects on patient morbidity but have no effect on mortality The misuse of antibiotic and the co-morbidities have direct negative effect in the response to medication and the progression of the disease Hypoglycemia is noticed more in intensive study.
\end{abstract}

Keywords: Hyperglycemia, Septic shock, Glycemic control.

\section{Introduction}

Sepsis, defined as a dysregulated host response to infection resulting in life-threatening organ dysfunction ${ }^{(1) .}$ The main pathophysiological feature of sepsis is the uncontrollable activation of both pro- and anti-inflammatory responses arising from the overwhelming production of mediators such as pro- and anti-inflammatory cytokines. Such an uncontrollable inflammatory response would cause many kinds of metabolic derangements. One such metabolic derangement is hyperglycemia [2].

Elevated blood sugar levels are commonly seen among critically ill patients, including those without a known history of diabetes. There are many reasons why patients undergoing treatment for critical illness develop hyperglycemia, and these reasons include both effects of endogenous stress responses and byproducts of medical interventions. Inflammatory cytokines and stress hormones, including cortisol and epinephrine, serve to inhibit insulin release and promote insulin resistance, thereby naturally increasing blood glucose levels by stimulating gluconeogenesis and glycogenolysis while impeding glucose uptake by peripheral tissues $[3,4]$.

Hyperglycemia is prevalent in critical care, caused by acomplex interaction of multiple feedback loops associatedwith inflammation as a result of immune responses,counter-regulatory responses, and high blood glucose itself $[5,6]$. Hyperglycemia is exacerbated by unsuppressed endogenous glucose production [7], some medications (steroids/ catecholamines), and high exogenously administered nutrition. There is also suppression/loss of pancreatic insulin secretion, and loss of sensitivity to insulin, resulting in reduced insulinmediated glucose uptake.Thus, the question arises of whether there is a need for an"artificial pancreas" or another form of closed-loop, highlypersonalized glycemic control (GC) in critical care, similar to those emerging in type 1 diabetes [8].

Since the very beginning of medicine the relation between glucose and illness has been of interest to physicians, as already Hippocrates stated: "Si quis febricitanti cibum det, convalescent quidem, robur: aegrotanti vero, morbus fit." (That nutrition, which is beneficial in the stage of convalescence from fever, would be truly injurious during the prevalence of the disease).More recent van Vught et al. have investigated the relation of admission hyperglycemia in patients suffering from sepsis [9]. In a sub-study of a prospective observational study they found that severe hyperglycemia $(>200 \mathrm{mg} / \mathrm{dL})$ but not mild hyperglycemia (141-199 $\mathrm{mg} / \mathrm{dL})$ at admission was associated with increased 30-day mortality. This was true for both patients with known diabetes and without diabetes, which is in contrast to previous findings by e.g., Stegenga et al. who reported an association of hyperglycemia with mortality only in patients without diabetes [10].

Of note, van Vught et al. further propose that the association of hyperglycemia and mortality is unrelated to exaggerated inflammation, endothelial cell activation and coagulation as severe hyperglycemia was associated with a decreased acute phase protein and cytokine response as well as an attenuated reduction in anticoagulant proteins such as protein $\mathrm{C}$ and antithrombin. This finding is surprising and new as it was thought and shown e.g., by Leonidou et al. that hyperglycemia is associated with increased proinflammatory cytokine production in septic patients [11].

In stress situations the body is thought to activate the central nervous system and neuroendocrine axes which release hormones such as catecholamines, glucagon and cortisol which are known to stimulate hepatic glucose production and lead to hyperglycemia 
[12]. Stress hyperglycemia is primarily caused by hepatic gluconeogenesis and glycogenolysis rather than by peripheral insulin resistance . Further, hyperglycemia is thought to be at least partially physiologic and reasonable for the organism from a survival standpoint [13].

Complications of diabetes contribute greatly to the increased mortality and morbidity associated with this disease. Diabetic complications are customarily divided into two main categories: macrovascular complications, including heart disease and stroke, and microvascular complications, which include retinopathy, nephropathy, and neuropathy [14].

\section{Patients and methods}

We included 60 patients divided into two groups..

Group1: consist of 30 patients was applied to intensive glycemic control.

Group 2: consist of 30 patients was applied to conventional glycemic control.

\subsection{Inclusion Criteria}

The study included patients who met the following conditions:

- Patients between 30 and 75 years old.

- Total leucocytic count $>12$ or $<4$ or $>10 \%$ bands.

- respiratory rate $>20$.

- heart rate $>90$.

- SBP $<90$ or MAP $<65$, or a SBP decrease of more than $40 \mathrm{mmHg}$ from the patient baseline.

- documentation of a suspected source of sepsis.

- Patients with serum lactate $>4 \mathrm{mmol} / \mathrm{L}$.

\subsection{Exclusion Criteria}

The study excluded patients who meet the following conditions:

- $\mathrm{GCS}=3$.

- bilateral tension pneumothorax.

- cardiac tamponade.

- hepatorenal syndrome.

- massive pulmonary embolism.

\subsection{Methods}

In a prospective, randomized, controlled study of adult patients admitted to our medical ICU, we will study patients who are considered to need intensive care for at least three days. On admission, patients will be randomly assigned to strict normalization of blood glucose levels ( 80 to $110 \mathrm{mg}$ per deciliter [4.4 to 6.1 mmol per liter]) with the use of insulin infusion or to conventional therapy (insulin administered when the blood glucose level exceeded $215 \mathrm{mg}$ per deciliter [12 mmol per liter], with the infusion tapered when the level fell below $180 \mathrm{mg}$ per deciliter [10 mmol per liter] (15). Data was collected from.

1. Demograghic data: ( age ,sex ,race ,socioeconomic status ,co-morbidities).

\section{Admission data}
A. Full history.
B. Clinical examination.
C. Full lab.
D. Chest $x$ ray.
E. Cultures blood sputum urine swab from wound.

3. We saw the outcome of that on:
A. Morbidity.
B. Mortality.
C. Time on mechanical ventilation.

\subsection{Statistical Methods}

Data management and statistical analysis were done using SPSS vs.25. (IBM, Armonk, New York, United States).

Numerical data were summarized as means and standard deviations. Categorical data were summarized as numbers and percentages.

Comparisons between both groups were done using the independent t-test for numerical data. Categorical data were compared using the Chi-square test or Fisher's exact test if appropriate.

Multivariate logistic regression analysis was used for the prediction of mechanical ventilation use and mortality. Odds ratios with $95 \%$ confidence intervals were calculated.

All $\mathrm{P}$ values were two-sided. $\mathrm{P}$ values less than 0.05 were considered significant.

\section{Results \\ This study was conducted on adult patients admitted to our medical ICU. Patients were divided into two groups: \\ Group I: Thirty patients applied to intensive glycemic control. \\ Group II: Thirty patients applied to conventional glycemic control. \\ Demographic characteristics in both groups \\ There were no significant differences between both groups as regard age and gender. \\ $P$ values were 0.219 and 1 , respectively.}

Table (1) Demographic characteristics in both groups.

\begin{tabular}{|c|c|c|c|c|}
\hline & & Group I $(n=30)$ & Group II $(n=30)$ & $P$ value \\
\hline Age & Mean \pm SD & $61 \pm 2$ & $59 \pm 7$ & 0.219 \\
\hline & Males $\quad n(\%)$ & $12(40.0)$ & $12(40.0)$ & 1.0 \\
\hline Gender & Females $\mathrm{n}(\%)$ & $18(60.0)$ & $18(60.0)$ & \\
\hline
\end{tabular}

The Independent t-test was used for age. Chi-square test was used for gender 


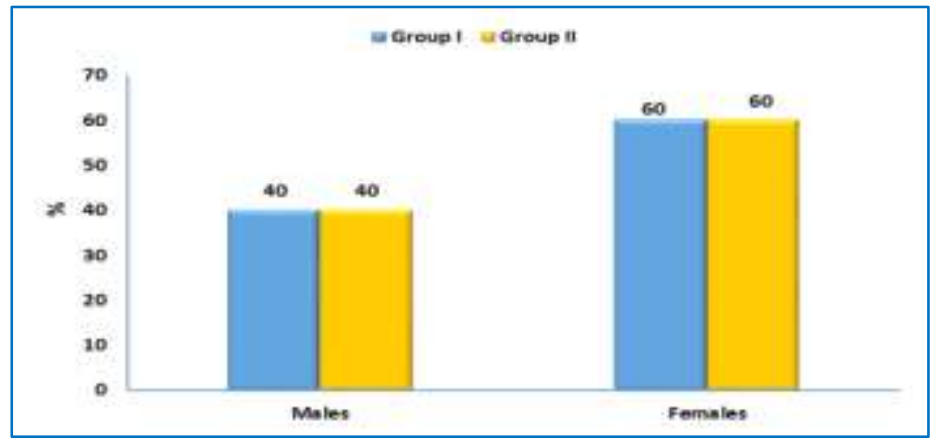

Fig. (1) Gender distribution in both groups.

\section{General characteristics in both groups}

There were no significant differences between both groups as regards all general characteristics

Table (2) General characteristics in both groups.

\begin{tabular}{lcccc}
\hline & & Group I $(\mathbf{n}=\mathbf{3 0})$ & Group II $(\mathbf{n}=\mathbf{3 0})$ & P value \\
\hline Shocked on support & $\mathbf{n}(\boldsymbol{\%})$ & $6(20.0)$ & $6(20.0)$ & 1.0 \\
DM & $\mathbf{n}(\%)$ & $24(80.0)$ & $24(80.0)$ & 1.0 \\
HTN & $\mathbf{n}(\%)$ & $10(33.3)$ & $12(40.0)$ & 0.592 \\
Asthmatic & $\mathbf{n}(\%)$ & $18(60.0)$ & $12(40.0)$ & 0.121 \\
Ischemic & $\mathbf{n}(\%)$ & $18(60.0)$ & $30(100.0)$ & $<0.001$ \\
Malignancy & $\mathbf{n}(\%)$ & $6(20.0)$ & $6(20.0)$ & 1.0 \\
CKD & $\mathbf{n}(\%)$ & $4(13.3)$ & $0(0.0)$ & 0.112 \\
\hline
\end{tabular}

Chi-square or Fisher's exact test was used

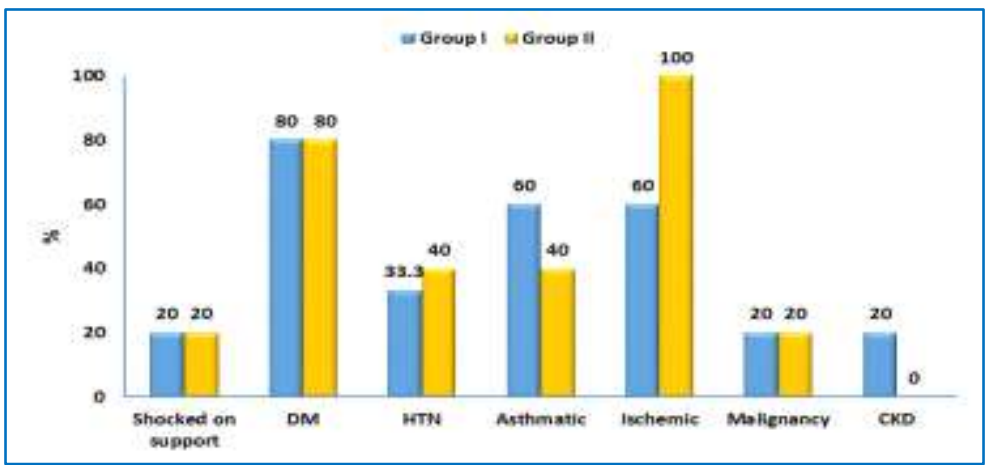

Fig. (2) General characteristics in both groups.

\section{Clinical findings in both groups}

Bedsores was significantly higher in group I (40\%) than group II $(0 \%)$. P-value was $<0.001$. There were no significant differences between both groups regarding chest infection, urinary tract infection, localized infection, leukocytosis, and leucopenia.

Table (3) Clinical findings in both groups.

\begin{tabular}{llccc}
\hline & & Group I(n= 30) & Group II (n= 30) & P value \\
\hline Chest infection & $\mathbf{n}(\%)$ & $12(40.0)$ & $18(60.0)$ & 0.121 \\
Urinary tract infection & $\mathbf{n}(\%)$ & $12(40.0)$ & $12(40.0)$ & 1.0 \\
Bedsores & $\mathbf{n}(\%)$ & $12(40.0)$ & $0(0.0)$ & $<0.001$ \\
Localized infection & $\mathbf{n}(\%)$ & $6(20.0)$ & $12(40.0)$ & 0.091 \\
Leukocytosis & $\mathbf{n}(\%)$ & $30(100.0)$ & $30(100.0)$ & NA \\
Leucopenia & $\mathbf{n}(\%)$ & $0(0.0)$ & $0(0.0)$ & NA \\
\hline
\end{tabular}

Chi-square test was used 


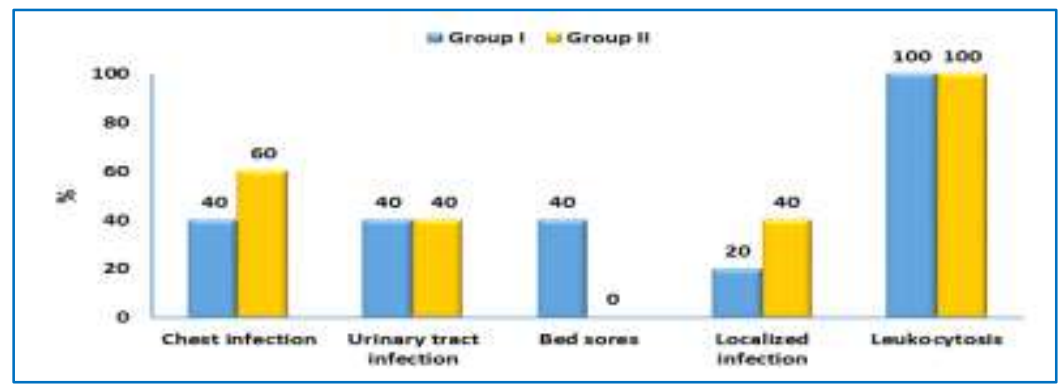

Fig. (3) Clinical findings in both groups.

\section{Morbidity outcome in both groups}

The renal shutdown was significantly higher in group II $(80 \%)$ than group I $(20 \%)$; P-value was $<0.001$. Recent ischemic events were significantly higher in group II (60\%) than group I $(0 \%)$; P value was $<0.001$.
Arrhythmia was significantly higher in group II (80\%) than group I (40\%); P-value was 0.002 . There were no significant differences between both groups as regard DVT and convulsions. P values were 0.091 for each.

Table (4) Morbidity outcome in both groups.

\begin{tabular}{lcccc}
\hline & & Group I $(\mathbf{n}=\mathbf{3 0})$ & Group II (n = 30) & P value \\
\hline Renal shut down & $\mathbf{n}(\boldsymbol{\%})$ & $6(20.0)$ & $24(80.0)$ & $<0.001$ \\
Recent ischemic events & $\mathbf{n}(\%)$ & $0(0.0)$ & $18(60.0)$ & $<0.001$ \\
Arrhythmia & $\mathbf{n}(\%)$ & $12(40.0)$ & $24(80.0)$ & 0.002 \\
DVT & $\mathbf{n}(\%)$ & $6(20.0)$ & $12(40.0)$ & 0.091 \\
Convulsions & $\mathbf{n}(\%)$ & $6(20.0)$ & $12(40.0)$ & 0.091 \\
\hline
\end{tabular}

Chi-square test was used

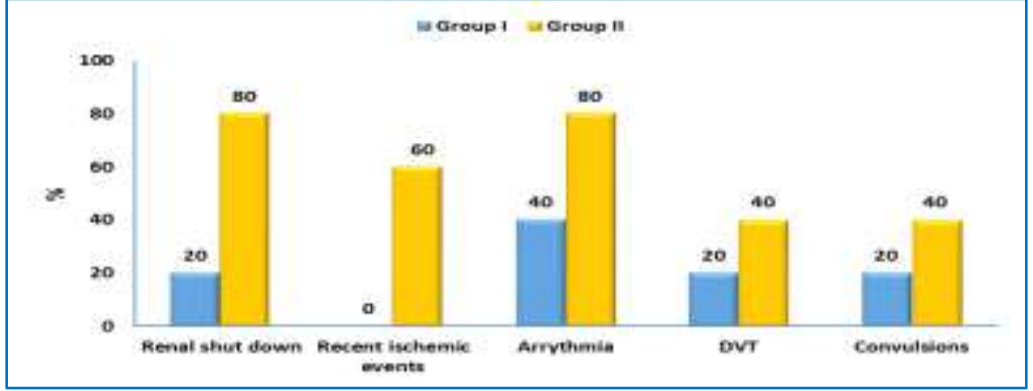

Fig. (4) Morbidity outcome in both groups.

\section{Mortality outcome in both groups}

There was no significant difference between both groups as regard mortality. P-value was 0.121

Table (5) Mortality outcome in both groups.

\begin{tabular}{lcccc}
\hline & & Group I $(\mathbf{n}=\mathbf{3 0})$ & Group II $(\mathbf{n}=\mathbf{3 0})$ & P value \\
\hline Mortality & $\mathbf{n}(\boldsymbol{\%})$ & $12(40.0)$ & $18(60.0)$ & 0.121 \\
\hline
\end{tabular}

Chi-square test was used

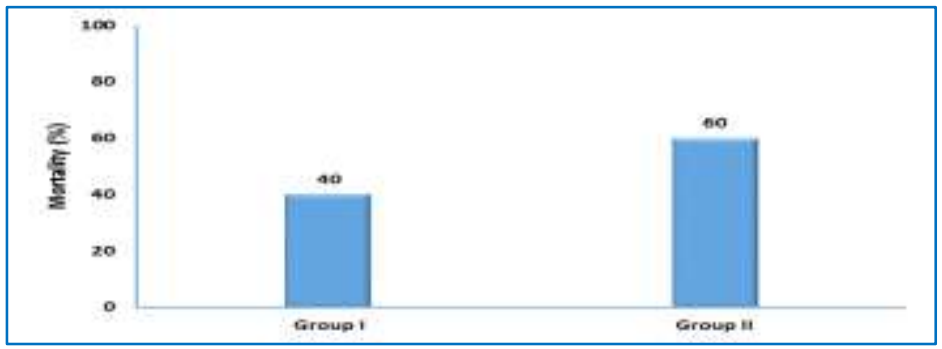

Fig. (5) Mortality outcome in both groups. 
Mechanical ventilation outcome in both groups

Use of mechanical ventilation was significantly higher in group II (80\%) than group I (40\%); P-value was 0.002 . There was no significant difference between both groups as regards the duration of mechanical ventilation. $\mathrm{P}$-value was 1 .

Table (6) Mechanical ventilation and its duration in both groups.

\begin{tabular}{lllccc}
\hline & & & Group I $(\mathbf{n}=\mathbf{3 0})$ & Group II (n= 30) & P value \\
\hline Mechanical ventilation & $\mathbf{n}(\boldsymbol{\%})$ & $12(40.0)$ & $24(80.0)$ & 0.002 \\
Duration of ventilation & Less than 1 week & $\mathbf{n}(\boldsymbol{\%})$ & $6(50.0)$ & $12(50.0)$ & 1.0 \\
& One to two weeks & $\mathbf{n}(\boldsymbol{\%})$ & $6(50.0)$ & $12(50.0)$ & \\
\hline
\end{tabular}

Chi-square test was used

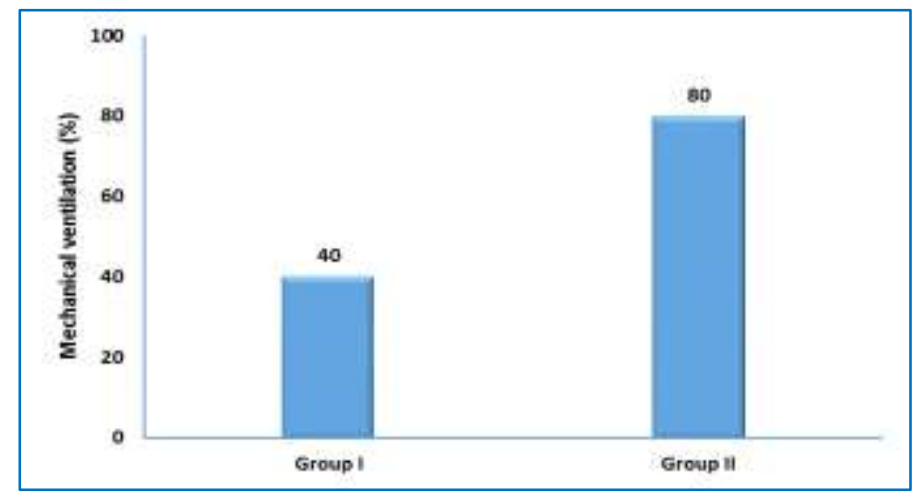

Fig. (6) Mechanical ventilation and its duration in both groups.

Duration of admission outcome in both groups

There was no significant difference between both groups as regards the duration of admission. P-value was 1.0

Table (7) Duration of admission in both groups.

\begin{tabular}{llccc}
\hline & & Group I (n= 30) & Group II (n= 30) & P value \\
\hline Duration of admission & Less than two weeks & $24(80.0)$ & $24(80.0)$ & 1.0 \\
& Two week to month & $6(20.0)$ & $6(20.0)$ & \\
\hline
\end{tabular}

Chi-square test was used

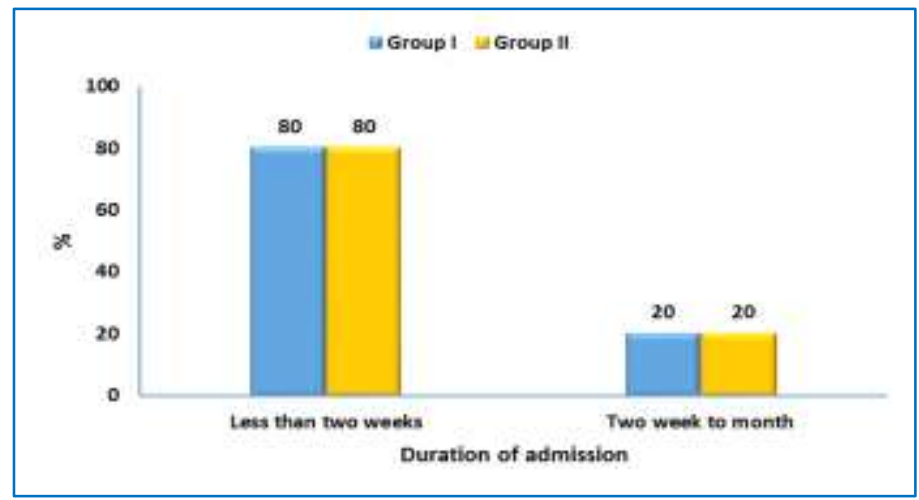

Fig. (7) Duration of admission in both groups.

Prediction of mechanical ventilation use and mortality

Multivariate logistic regression was done for prediction of mechanical ventilation use and mortality. Intensive therpay was significantly associated with $88.7 \%$ risk reduction of mechanical ventilation use (OR
$=0.113,95 \% \mathrm{CI}=0.03-0.423 \& \mathrm{P}$ value $=0.001) . \mathrm{As}$ regard mortality, intensive therapy was borderline significantly associated with $65.3 \%$ risk reduction of mortality $(\mathrm{OR}=0.347,95 \% \mathrm{CI}=0.115-1.048 \& \mathrm{P}$ value $=0.061)$. 
Table (8) Multivariate logistic regression analysis for prediction of MV use and mortality.

\begin{tabular}{|c|c|c|c|c|}
\hline & \multicolumn{2}{|c|}{ Mechanical ventilation use } & \multicolumn{2}{|c|}{ Mortality } \\
\hline & OR $(95 \% \mathrm{CI})$ & P-value & OR $(95 \% \mathrm{CI})$ & P-value \\
\hline Intensive therapy* & $\begin{array}{c}0.113 \\
(0.03-0.423)\end{array}$ & 0.001 & $\begin{array}{c}0.347 \\
(0.115-1.048)\end{array}$ & 0.061 \\
\hline
\end{tabular}

\section{Discussion}

Sepsis is dysregulated host response to infection resulting in life-threatening organ dysfunction. Elevated blood sugar levels are commonly seen among critically ill patients, including those without a known history of diabetes

Since the beginning of medicine the relation between glucose and illness has been of interest to physicians.

Elevated blood sugar levels are commonly seen among critically ill patients, including those without a known history of diabetes. There are many reasons why patients undergoing treatment for critical illness develop hyperglycemia, and these reasons include both effects of endogenous stress responses and byproducts of medical interventions. Inflammatory cytokines and stress hormones, including cortisol and epinephrine, serve to inhibit insulin release and promote insulin resistance, thereby naturally increasing blood glucose levels by stimulating gluconeogenesis and glycogenolysis while impeding glucose uptake by peripheral tissues.

The start of this study In 2001 when van den berge noticed the effect of glycemic control in patients with septic shock and reported that intensive glycemic control has more favroubable outcome in morbidity and mortality in surgical ICU and made astudy on medical icu but found that intensive glycemic control have better outcome in morbidity but not mortality.

Complications of diabetes contribute greatly to the increased mortality and morbidity associated with this disease. Diabetic complications are customarily divided into two main categories: macrovascular complications, including heart disease and stroke, and microvascular complications, which include retinopathy, nephropathy, and neuropathy

The main value of glycemic control is sepsis is to prevent or delay the occurrence of multiorgan dysfunction and/or developing complication.

Our study on tight versus glycemic control in patient with sepsis and septic shock 30 patients were applied to tight glycemic control and 30 were applied to conventional glycemic control in Medical ICU in benha university hospital.

Compared to van den berge study published in new England journal of medicine 2001 patients mean age in group 1 was 63.4 years and 62.3 years in group 2 .

In our study mean age in group 1 was 61 years and 59 years in group 2 .

In van den berge study history of cancer in group 1 was $16 \%$ and $15 \%$ in group 2 .

$20 \%$ of patients have history of cancer in group 1 and $20 \%$ in group 2 in our study.
In van den berge study $4.8 \%$ in group 1 and $8.2 \%$ in group 2 exposed to renal shutdown.

In our study patients who were exposed to renal shutdown was $20 \%$ in group 1 and $80 \%$ in group 2 .

both studies are clinically insignificant.

$7.5 \%$ in group 1 and $11.9 \%$ in group 2 needed mechanical ventilation which is statistically significant

In our study $40 \%$ of patients in group 1 and $80 \%$ of patients in group 2 needed mechanical ventilation which is statistically highly significant

In van den berge study mortality rate in group 1 was $8 \%$ and $4.6 \%$ in group 2 which is statistically significant

Mortality rate in our study $60 \%$ in group 1 and $80 \%$ in group 2 which is statistically insignificant

Frank M Brunkhorst studied 537 patients with mean age 65.2 years in group 1 and 64 years in group 2 .

In Frank M Brunkhorst percentage of cancer $8.9 \%$ in group 1 and $9.3 \%$ in group 2

Our study showed percentage of cancer $20 \%$ in group 1 and $20 \%$ in group 2

Both studies are clinically insignificant.

In Frank $M$ Brunkhorst study Mean number of hospital stay is $16 \%$ in group 1 and $14 \%$ in group 2 which is statistically insignificant.

In our study 24 patinets stayed in hospital for less than 2 weeks and 6 patients stayed for 2 weeks to amonth in both groups which is statistically insignificant.

In Frank M Brunkhorst study $27.5 \%$ of patients in group 1 and $22.5 \%$ of patients in group 2 need hemodialysis which is statistically insignificant.

In our study $20 \%$ in group 1 needed hemodialysis and $80 \%$ of patients required hemodialysis in group 2 which is statistically highly significant.

In Frank M Brunkhorst study $12 \%$ in group 1 and $40 \%$ of patients in group 2 of patients have hypoglycemia.

$20 \%$ of patients in group 1 and $60 \%$ of patients in group 2 in our study most of hypoglycemic episodes occur at night due to less nursing staff follow up.

NICE SUGAR trial was on mean age group 60.4 years on group 1 and 59.9 on group 2 .

In our study mean age of group 1 was 61 years and 59 years in group 2.

$93 \%$ was ventilated in group 1 and 92.7 was ventilated in group 2 which is statistically insignificant.

In our study which revealed $40 \%$ in group 1 and $80 \%$ in group 2 require mechanical ventilation which is statistically significant.

On day $90.27 .5 \%$ of patients exposed to strict glycemic control died versus $24.9 \%$ of patients in group 2 died which is statistically significant . 
On day $2822.3 \%$ of patients applied to strict study was died and $20.8 \%$ applied to conventional glycemic control was died which is statistically insignificant.

In our study $40 \%$ of patients applied to tight glycemic control died and $80 \%$ was died applied to conventional glycemic control which is statistically insignificant .

In NICE SUGAR trial $96 \%$ of group 1 was ventilated and $95.3 \%$ was ventilated in group 2 which is statistically insignificant.

In our study $40 \%$ of patients in group 1 was ventilated and $80 \%$ of patients in group 2 was ventilated which is statistically significant .

$14.5 \%$ of patients in group 1 require hemodialysis and $15.4 \%$ in group 2 which is statistically insignificant.

In our study $20 \%$ in group 1 needed hemodialysis and $80 \%$ of patients required hemodialysis in group 2 which is statistically highly significant.

Our study compared to the others and similarity and the difference between them is due to various factors firstly the stage of the disease . once the patient became in shock stage he become critical. Van den berge study and van bunkroust studies the effect of normalization of blood glucose in surgical patients with less comorbidities and more fit to overcome sepsis . in Europe medical and surgical facilities more, life style better, soscioeconomic status better, environment and food more healthy, mean age of death in general higher than ours also one of the most common problems that face us in study that in most cases who need urgent surgical intervention is delayed or cancelled delayed to reasonable reasons or for fear of medicolegality.

One of the most obstcales in the progression of septic shock is the misuse of antibiotic to adegree that culture and sensitivity test become resistant to most antibiotics

\section{Conclusion}

- We found that intensive glycemic control have more favourable effects on patient morbidity but have no effect on mortality

- The misuse of antibiotic and the co-morbidities have direct negative effect in the response to medication and the progression of the disease

- Hypoglycemia is noticed more in intensive sudy

\section{Recommendation}

- we recommend tight glycemic control in patients with septic shock

- strict follow up of glycemic control

- follow sepsis care bundles on admission

\section{References}

[1] M.Singer, CS.Deutschman, CW.Seymour. The Third International Consensus Definitions for Sepsis and Septic Shock (Sepsis-3). JAMA.vol.15,pp.801,2016.

[2] I.Cinel, SM.Opal. Molecular biology of inflammation and sepsis: a primer. Crit Care Med.vol.37,pp.291304,2015 .
[3] D.Lena, P. Kalfon, JC.Preiser ,C. Ichai. Glycemic control in the intensive care unit and during the postoperative period. Anesthesiology.vol.114,pp.438-444,2011.

[4] BP.Kavanagh KC.McCowen,Clinical practice. Glycemic control in the ICU. N Engl J Med.vol.363,pp.2540-2546,2010.

[5] KM .Dungan ,SS.Braithwaite, JC.Preiser. Stress hyperglycaemia. Lancet.Vol. 373,pp.1798-1807. doi: 10.1016 /S0140-6736 (09)60553-5,2009.

[6] PE.Marik, M.Raghavan. Stress-hyperglycemia, insulin and immunomodulation in sepsis. Intensive Care Med.vol.8,PP.748-756. doi: 10.1007 / s00134004-2167-y, 2004.

[7] TW. Rice. Gluttony in the intensive care unit: time to push back from the consensus table. Am J Respir Crit Care Med.vol.187,pp.223-224. doi: 10.1164/rccm.201212-2228ED, 2013.

[8] E. Renard, A .Farret ,J. Kropff, D.Bruttomesso, M.Messori ,J.Place. Day-and-night closed-loop glucose control in patients with type 1 diabetes under free-living conditions: results of a single-arm 1month experience compared with a previously reported feasibility study of evening and night at home. Diabetes Care.vol.39,PP.1151-1160. Doi10.2337/dc16-0008,2016.

[9] LA. van Vught, MA.Wiewel, PM.Klein Klouwenberg. Admission Hyperglycemia in Critically Ill Sepsis Patients: Association With Outcome and Host Response. Crit Care.vol.44,PP.1338-46,2016.

[10] ME. Stegenga, JL. Vincent ,GM. Vail, et al. Diabetes does not alter mortality or hemostatic and inflammatory responses in patients with severe sepsis. Crit Care .vol.38:539-45. 2010,

[11] GC. Koh, SJ .Peacock, T. van der Poll. The impact of diabetes on the pathogenesis of sepsis. Eur J Clin Microbiol Infect Dis.vol.31,pp.379-88,2012.

[12] M.Oddo, JM.Schmidt, E. Carrera. Impact of tight glycemic control on cerebral glucose metabolism after severe brain injury: a microdialysis study. Crit Care Med.vol.36,pp.3233-8, 2008.

[13] PE.Marik, R.Bellomo. Stress hyperglycemia: an essential survival response! Crit Care.vol.17,pp.305,2013.

[14] L.Litwak, S. Y.Goh , Z.Hussein, R .Malek, V.Prusty, M. E. Khamseh Prevalence of diabetes complications in people with type 2 diabetes mellitus and its association with baseline characteristics in the multinational A1chieve study. Diabetology and Metabolic Syndrome.vol.5(1),pp.57. doi: 10.1186 / 1758-5996-5-57,2013.

[15] MS. Agus, GM. Steil, D .Wypij, JM. Costello, PC .Laussen, $M$.Langer, JL .Alexander, LA. Scoppettuolo, FA .Pigula, JR. Charpie. Tight glycemic control versus standard care after pediatric cardiac surgery. N Engl J Med.vol.367(13),pp.12081219.doi: 10.1056/NEJMoa1206044,2014. 